\title{
Fast derivation of soil surface roughness parameters using multi-band SAR imagery and the Integral Equation Model
}

\author{
Benjamin Seppke, Leonie Dreschler-Fischer, Jo-Ann Heiming, Felix Wengenroth \\ University of Hamburg, MIN Faculty, Dept. of Informatics, Cognitive Systems Laboratory \\ \{seppke, dreschler, lheiming, 3wengenr\}@informatik.uni-hamburg.de
}

\begin{abstract}
The Integral Equation Model (IEM) predicts the normalized radar cross section (NRCS) of dielectric surfaces given surface and radar parameters. To derive the surface parameters from the NRCS using the IEM, the model needs to be inverted. We present a fast method of this model inversion to derive soil surface roughness parameters from synthetic aperture radar (SAR) remote sensing data. The model inversion is based on two different collocated SAR images of different bands, the derivation of the parameters cannot be done using one band alone. The computation of the model and the model inversion are very time consuming tasks and therefore may be impractical for large remote sensing data. We present an approach that is based on a few model assumptions to speed up the computation of the surface parameters. We applied the algorithm to detect the correlation length of the surface for dry-fallen areas in the World Cultural Heritage "Wadden Sea”, a coastal tidal flat at the German Bight (North Sea). The results are very promising and may be used for a classification of the area in future steps.
\end{abstract}

\section{Introduction}

The derivation of soil surface roughness parameters using remote sensing data plays an important role in the classification of these areas. It is often the first necessary step that has to be performed, before the classification can be done using expert's knowledge about the surface parameters. In this paper, we will show how multi-band SAR images (see [5]) can be used for a fast derivation of the surface roughness parameters.

The radar backscattering of soil surface is measured by the SAR sensors and depends on different parameters: the soil's dielectric constant, surface roughness features, etc. Thus, the captured radar backscattering can be seen as a combination of these parameters, which makes it difficult to compute a surface parameter from radar backscatter. However, Gade et al. have shown, that the Integral Equation Model (see [1]) can be used to estimate soil surface roughness by means of root mean square (rms) height and correlation length, directly from a pair of dual frequency SAR images if a proper backscatter model is used (see [3]). This conclusion holds for tidal flat areas, which are coastal areas that fall dry once during each tidal cycle.

In 1994, aboard the space shuttle "Endeavour" was a multi-frequency (C- and X-band) SAR sensor mounted (the SIR-C/X-SAR) that simultaneously imaged the same area using different radar wavelengths. Although this was a valueable configuration of sensors, it has never been rebuild. Hence we have to use at least two single sensor SAR satellites for the derivation of surface parameters. We have chosen images of the ALOS PALSAR (L-band) and the ENVISAT ASAR (C-band) sensors for this paper.

The derivation of the surface parameters is achieved by inverting the IEM. The direct application of the model and the model inversion are of high computational complexity. We will introduce the IEM in the next chapter and show possibilities to increase the speed of the computation of surface roughness features.

\section{The Integral Equation Model}

The IEM predicts the NRCS of bare soil as a function of its dielectric constant and surface roughness. The latter is generally described by the autocorrelation function and the standard deviation of the roughness height (rms height). According to Fung et. al (see [1] [2]) the single-scattering term of the IEM for vertical (VV) polarization, $\sigma_{v v}^{0}$, can be expressed as:

$$
\sigma_{v v}^{0}=\frac{k^{2}}{2} \exp \left(-2 k_{z}^{2} h^{2}\right) \sum_{n=1}^{\infty} h^{2 n}\left|I_{v v}^{n}\right|^{2} \frac{W^{n}\left(-2 k_{x}, 0\right)}{n !}
$$


where

$$
\begin{aligned}
& I_{v v}=\quad\left(2 k_{z}\right)^{n} \frac{2 R_{\|}}{\cos (\theta)} \exp \left(-h^{2} k_{z}^{2}\right) \\
& +\frac{k_{z}^{n}\left[F_{v v}\left(-k_{x}, 0\right)+F_{v v}\left(k_{x}, 0\right)\right]}{2},
\end{aligned}
$$

$k$ is the radar wavenumber that can be divided into the ground range component $k_{x}$ and the vertical component $k_{z}$, and $h$ denotes the rms surface height. The function $W^{n}$ denotes the Fourier transform of the $n$-th power of the surface autocorrelation function, and $\theta$ is the radar incidence angle. $R_{\|}$is the Fresnel reflection coefficient for parallel polarization (i.e., in the plane of incidence), and $F_{v v}$ is a field coefficient for vertically polarized scattering, which described in more detail by Fung et al. in [1]. Note that the definition for the horizontal ( $\mathrm{HH})$ polarization follows directly from Eq. 1. For this paper we use the updated version of the Fresnel coefficients as proposed in [2].
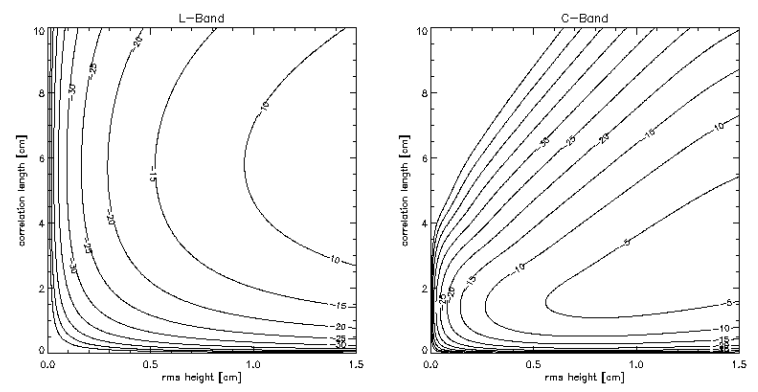

Figure 1. Isolines derived by the IEM

The graphs show the dependencies between the NRCS (dB) value (plotted isolines), the correlation length, and the rms height for both used bands.

To determine the surface autocorrelation function, in-situ measurements using a sledge-mounted sensor have been carried out by Tanck [6]. The results of these measuments indicated that the surface autocorrelation function of tidal flats is very similar to a Gaussian autocorrelation function, hence we use this autocorrelation function for the calculation of the rms height and the correlation length. Fig. 1 shows the resulting isolines for the L- and C-band respectively.

\subsection{Model inversion}

The IEM describes the NRCS in dependency to the rms height and the correlation length. For the task of deriving surface roughness parameters, Gade et. al proposed to invert the model (see [3]). As each measured NRCS value represents one isoline in the correlation length / rms-height space, a direct estimation of both parameters cannot be done with one SAR image alone (see Fig. 1, [6]).

To perform the inversion, we use the multi-sensor (multi-frequency) approach of two collocated SAR images proposed by Gade et. al. (see [3]). The inversion is performed in two steps. The first step is the assignment of each pixel's NRCS value to a corresponding isoline of the IEM. The second step is the determination of the intersection point, which yields to the surface parameters (see [3]).

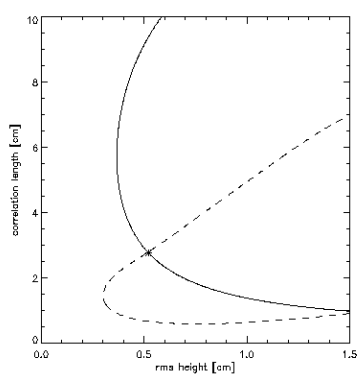

\section{Figure 2. Inversion of the IEM}

The graphs shows the $-18 \mathrm{~dB}$ L-band isoline (solid) together with the $-9 \mathrm{~dB} C$-band isoline (dashed). The intersection point with the lowest rms height (cross) is used for the model inversion.

The example of Fig. 2 shows the two isolines (calculated with the IEM) that correspond to real NRCS values derived from collocated pixels in the two SAR images of a selected position on the tidal flats. The model inversion for this example results leads to a correlation length of $2.78 \mathrm{~cm}$ and a rms height of $0.52 \mathrm{~cm}$.

It should be mentioned that the isolines for $\mathrm{C}$ - and $\mathrm{X}$-band are quite similar because of the similar radar wavelength compared to those for L-band. Therefore, Gade et al. suggest that L-band images in combination with either C- or X-band images are best suited for deriving soil surface roughness parameters using the IEM inversion method (see [3]). Although the X-band is not used for this study, it can be shown that the intersection point is near to the marked intersection of Fig. 2 (see [6]). Further, it is beneath the intersection with the lower rms height. Thus, we have chosen this intersection point between the $\mathrm{L}$ - and C-band if there is more than one intersection of both isolines.

\subsection{Speed enhancement of the model inversion}

Building up the model space and running the model inversion for a mega- of giga-pixel satellite image is a very time-consuming task. Each pixel has slightly different (angular) model parameters, which means that 
the full model space has to be determined once per pixel. Even if the model spaces could be precalculated, it would still be time-consuming to find the intersection of the isolines that is used for the model inversion (see Fig. 2). To increase the speed of the model inversion we propose the following computational optimizations:

In satellite radar imagery the incidence angle of the radar beam changes across the radar image swath, it increases from near-range to far-range. In our calculations we make use of the incidence angle in the calculation by the IEM. To investigate the influence of incidence angle distribution in derivation of the NRCS we compared the derivation of exact angle with an average value of incidence angle. We found out that the worst case error is less than $0.3 \mathrm{~mm}$ for correlation length and $0.03 \mathrm{~mm}$ for rms height. For our needs the use of an average angle is sufficient. This allows the same set of model parameters for SAR images and thus to pre calculate the model space of a pair of images.

Instead of searching for an intersection between the isolines of the models for each pixel, we create a priori random access tables. These tables store the rms height and the correlation length for two given NRCS values. Note, that each table is only valid for one band and angle configuration. However, the angles used by the satellite are fixed to a few configurations for each satellite. We further have to discretize the domain to allow only NRCS values within a certain interval and at a certain resolution between each value. To meet qualitative requirements of the oceanographers, we found out that a domain (NRCS) discretization for the range of $[-29.8,0]$ using a resolution of $0.2 \mathrm{~dB}$ is sufficient. The average differences in the results are less than $0.5 \mathrm{~cm}$ for correlation length and $0.5 \mathrm{~mm}$ for $\mathrm{rms}$ height. This results in table sizes of $150 \times 150=22,500$ entries. The table creation still is a time consuming operation, but it leads to re-usable tables on the one hand and to a nearly constant time complexity for the derivation of correlation length and rms height on the other hand, given two NRCS values. A brief comparison of the computation times is given in Tab. 2.

\section{Results}

For the derivation of the soil surface roughness parameters, we have selected a pair of images, that were taken by the ALOS PALSAR and ENVISAT ASAR. To derive the surface parameters of the dry-fallen areas of tidal flats, we have to ensure that the images have been recorded during the same (low) tide conditions. This is the case for the selected images as they were captured about half an hour after the local low tide. More information about the images can be found in Tab. 1 resp.
Fig. 3 for the region of interest.

Table 1. Satellite Image Characteristics

\begin{tabular}{|l|c|c|}
\hline & $\begin{array}{c}\text { ALOS } \\
\text { PALSAR }\end{array}$ & $\begin{array}{c}\text { ENVISAT } \\
\text { ASAR }\end{array}$ \\
\hline Date & $08 / 04 / 1221: 43$ & $08 / 04 / 1310: 01$ \\
\hline Polarisation & $\mathrm{H} / \mathrm{H}$ & $\mathrm{V} / \mathrm{V}$ \\
\hline Band & $\mathrm{L}(\lambda=235 \mathrm{~mm})$ & $\mathrm{C}(\lambda=58 \mathrm{~mm})$ \\
\hline Angle & $38.7^{\circ}$ & $22.5^{\circ}$ \\
\hline Resolution & $6.25 \mathrm{~m} / \mathrm{px}$ & $12.5 \mathrm{~m} / \mathrm{px}$ \\
\hline
\end{tabular}

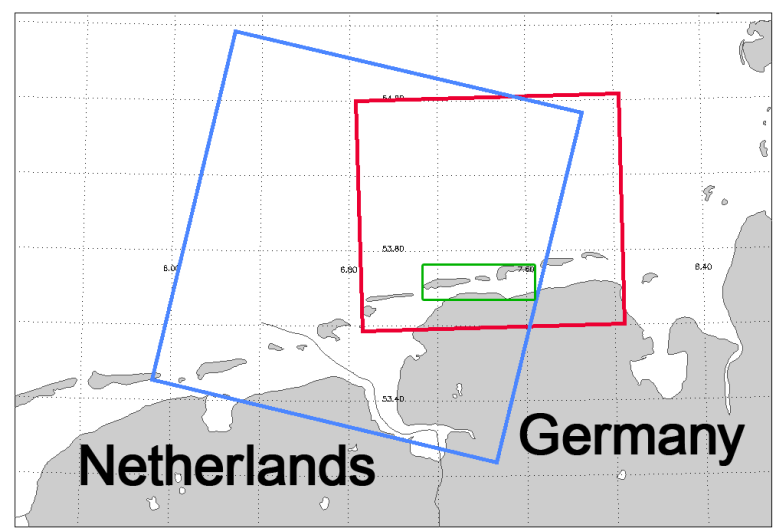

Figure 3. Location of images and ROI

The large blue region denotes the position of ENVISAT ASAR image, the red one corresponds to the PALSAR image. The ROI is shown by the green frame.

For the model inversion, we precalculated the random access tables for both sensor angles using the parameters given in Tab. 1. Before applying the model inversion using both images, we register the images to same size and geographic area, and filter both using a Gamma Maximum A Posteriori (MAP) filter with a kernel-size of $11 \times 11$ pixel (see [4]). This preprocessing is necessary to suppress the speckle noise that occurs in SAR images (see [5]). We also mask out areas of water and land manually, based on the ENVISAT image (see Fig. 4 topmost panel). Fig. 4 depicts the images of both sensors for the selected region of interest (ROI).

The model and the model inversion has been implemented in IDL. The times in Tab. 2 have been taken on an Apple iMac with a Core2Duo $2.83 \mathrm{GHz}$ CPU and 4 GB of RAM. Note that the time of finding an intersection point varies due to the properties of the model space. For the times per megapixel in Tab. 2 we have used the mean value for this search operation, which is about 372 milliseconds per intersection.

Fig. 4 shows the results of the model by means of the 
Table 2. Time complexity

\begin{tabular}{|l|r|}
\hline & Processing time [s/MP] \\
\hline Normal model inversion & 14372093 \\
\hline Fixed angle inversion & 512093 \\
\hline Creation of access tables & 8386 \\
\hline Access of surf. param. & 5 \\
\hline
\end{tabular}

derived correlation length. During the model inversion, some pixel did not lead to an intersection of the isolines for L- and C-band (see Fig. 2) and thus we could not derive a valid correlation length for these locations. They are diplayed as white spots in Fig. 4. We did not perform an interpolation over these areas, because it is not yet clear what caused the unreliable NRCS values for both sensors at the region of interest.

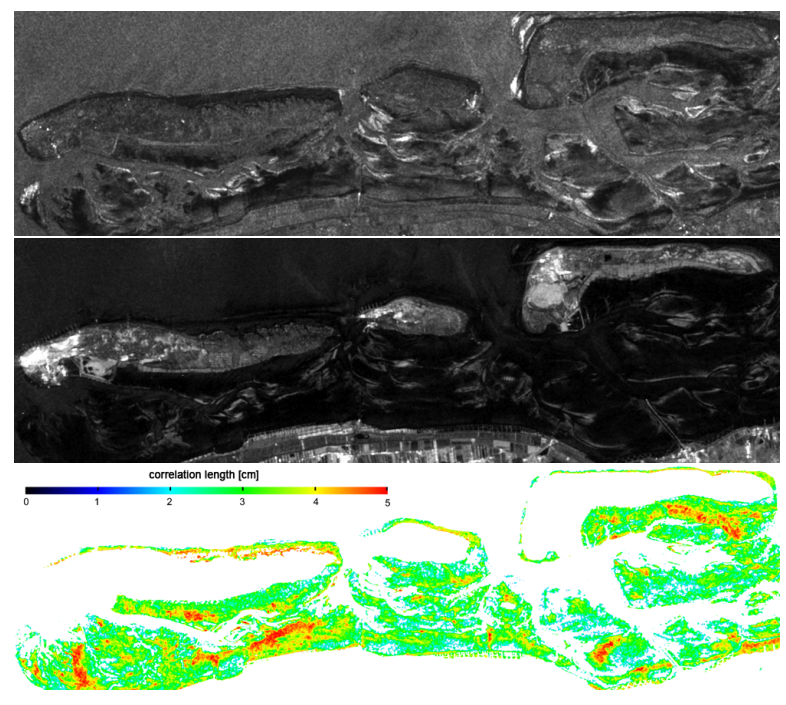

Figure 4. Images and results (ROI)

The upper image depicts the filtered ASAR image, whereas the second image depicts the filtered PALSAR image of the selected ROI. Both have been contrast enhanced for display purpose.The bottom image shows the derived correlation length for the ROI. Note that islands, land, and water have been masked out before the calculation. Data provided by the European Space Agency and by the German Aerospace Center.

\section{Conclusions}

We have presented an efficient approach to derive soil surface roughness parameters using multi-band SAR imagery and the integral equation model. For the derivation, we need to invert the IEM that predicts the NRCS for given surface roughness parameters.
The model itself is of a high computational complexity, and this complexity even increases when the inversion needs to be computed. To achieve to a lower complexity, we made some model assumptions and used precalculated random access tables that allow for a fast derivation of the surface parameters.

We have shown that the assumption of a fixed angle over the whole scene and the calculation of randomaccess tables leads to an increased algorithm speed, without losing much of its precision. Our proposed algorithm is about 1700 times faster compared to the standard implementation of the model and its inversion (see Tab. 2). This allows for a derivation of soil surface roughness parameters using high resolution SAR imagery in future.

Note that our implementation in IDL may not be the fastest one, since IDL does not optimize all user-written functions during compilation to reach the performance of other programming languages like $\mathrm{C}$. We will therefore explore implementations of the proposed algorithm in other programming languages in future.

\section{Acknowledgements}

The authors are grateful to Martin Gade for the help in allocating and analyzing the data sets of the ALOS PALSAR and ENVISAT ASAR satellite.

This work is partly supported by the German national project DeMarine (50 EE 0817).

\section{References}

[1] A. K. Fung and K. S. Chen. Backscattering from a Randomly Rough Dielectic Surface. IEEE Transactions on Geoscience and Remote Sensing, 30:356-369, 1992.

[2] A. K. Fung and K. S. Chen. An update on the IEM surface backscattering model. IEEE Geoscience and Remote Sensing Letters, 1:75-77, 2004.

[3] M. Gade, W. Alpers, C. Melsheimer, and G. Tanck. Classification of sediments on exposed tidal flats in the German Bight using multi-frequency radar data. Remote Sensing of Environment, 112(4):1603-1613, April 2008.

[4] A. Lopes, E. Nezry, R. Touzi, and H. Laur. Structure detection and statistical adaptive speckle filtering in SAR images. International Journal of Remote Sensing, 14(9):1735-1758, 1993.

[5] J. Richards. Remote Sensing with Imaging Radar. Springer, 2009.

[6] G. Tanck. Untersuchung der Radarrückstreueigenschaften unterschiedlicher Wattypen des schleswig-holsteinischen Wattenmeeres mit Hilfe eines Multifrequenz/Multipolarisations-SAR. Diploma thesis, Fachbereich Physik, Universität Hamburg, Hamburg, Germany, August 1998. 